\title{
Signatures of molecular structure in the strong- field response of aligned molecules
}

\author{
M. LEIN ${ }^{1}$, R. DE NALDA ${ }^{2}$, E. HEESEL ${ }^{3}$, N. HAY $^{3}$, \\ E. SPRINGATE ${ }^{3}$, R. VELOTTA ${ }^{4}$, M. CASTILLEJO ${ }^{5}$, \\ P. L. KNIGHT ${ }^{3}$ and J. P. MARANGOS ${ }^{3}$ \\ ${ }^{1}$ Max Planck Institute for the Physics of Complex Systems, Nöthnitzer \\ Straße 38, 01187 Dresden, Germany \\ ${ }^{2}$ Dpto. Química Física I, Facultad de Ciencias Químicas, Universidad \\ Complutense de Madrid, Avda. Complutense s/n, 28040 Madrid, Spain \\ ${ }^{3}$ Blackett Laboratory, Imperial College London, Prince Consort Road, \\ London SW7 2BW, UK \\ ${ }^{4}$ Istituto Nazionale Fisica della Materia, Dipartimento Scienze Fisiche, \\ Via Cintia 26, 80126 Napoli, Italy \\ ${ }^{5}$ Instituto de Química-Física Rocasolano, Serrano 119, 28006 Madrid, \\ Spain
}

(April 1, 2004)

\begin{abstract}
The strong-field response of molecules exhibits interference effects due to the geometric and electronic structure of the molecules and provides a basis for ultrafast imaging of molecular structure. This is demonstrated for high-order harmonic generation and high-order abovethreshold ionization in aligned molecules by numerical solution of the time-dependent Schrödinger equation. Experimental and theoretical results for high-order harmonic generation with aligned $\mathrm{CO}_{2}$ molecules show that the harmonics exhibit an orientation dependence that is explained by the valence orbital symmetry. A detailed discussion of phasematching effects due to the presence of different molecular orientations in an ensemble of imperfectly aligned molecules is presented.
\end{abstract}

\section{Introduction}

Within the field of laser science, there are currently two major approaches to the time-resolved measurement of processes on time scales below $1 \mathrm{fs}$. First, one can utilise attosecond light pulses from high-order harmonic generation (HHG) to start fast photoinduced processes that are then probed with a synchronised laser light 
wave [1]. Alternatively, the detailed knowledge about the mechanisms of strongfield processes allows to extract information about the time evolution only from the response to an intense laser [2]. This method does not involve attosecond light pulses. Rather, it exploits the presence of attosecond electron wave packets that are created during the interaction with the laser. These wave packets are associated with electrons that are initially stripped off the atom or molecule by the laser and are driven back to the core a short time later. These recollisions give rise to various phenomena including HHG, double ionization and high-order above-threshold ionization (high-order ATI) [3, 4].

This work focuses on the question of how information about the molecular structure is imprinted on the measurable recollision products, in particular high harmonics and photoelectrons. From the analogy to electron diffraction with an external electron beam, it appears plausible that interference patterns due to the molecular structure can be observed in such a 'self-diffraction' setup. Section 2 gives a brief review of numerical calculations $[5,6,7,8]$ demonstrating that these interferences are indeed found for high harmonics and high-energy photoelectrons.

The diffraction-type effects should be distinguished from interferences occurring not in the recollision, but in the ionization process itself. These have been analysed previously [9], and it appears that for example the suppression of ionization in $\mathrm{O}_{2}$ molecules is due to destructively interfering contributions from the two $\mathrm{O}$ atoms.

In any case, since molecules are not isotropic, we expect that the response to the laser depends on the orientation of the molecules. For linear molecules and linear laser polarization, the orientation is simply described by the angle between the molecular axis and the electric field. Early theoretical papers have studied the orientation dependence of HHG [10]. Later the problem was reinvestigated in the context of interference effects $[5,6,7]$. After pioneering experimental work on HHG with aligned molecules that investigated only the cases of orientations parallel and perpendicular to the field [11], the orientation dependence is now investigated in more detail by several workers $[12,13]$. Our experimental and theoretical results for the case of $\mathrm{CO}_{2}$ molecules are discussed in section 3 .

Clearly, in order to deduce information about the molecular structure, it is desirable to work with aligned molecules rather than a randomly oriented ensemble. However, perfect alignment can never be reached, so the measured harmonics always represent a coherent sum of the contributions from different orientations. If the phases of the harmonics vary with orientation, phase mismatch will lead to a reduction of the harmonic signal. Theoretically, this sort of phase matching has only been studied for completely randomly oriented molecules so far [14]. In section 4, we give a generalised discussion for partially aligned distributions of arbitrary width. 


\section{Interferences in HHG and high-order ATI}

The physics of laser-driven electron recollision in a two-centre molecule is illustrated in figure 1. Initially, the system is singly ionized and the emerging electron is accelerated in the direction opposite to the electric field. When the sinusoidal laser field $E(t)=E_{0}(t) \sin \left(\omega_{\mathrm{L}} t\right)$ reverses its direction, the electron is accelerated back towards the core. The recollision of the electron may (besides other possibilities) lead to recombination or elastic scattering. In the first case, a high-harmonic photon is generated while in the second case the electron is further accelerated by the laser and escapes as a fast photoelectron.

The electron wave packet spreads during its motion after ionization and quickly becomes much larger than the molecule. It is therefore helpful to think of the recollision in terms of a plane wave with wave vector $\mathbf{k}$ impinging on the core. If the electron recombines, it will end up in a two-centre molecular orbital, and the corresponding two contributions to the emission spectrum will add coherently. Similarly, in the case of scattering, the electron can scatter from either centre, and the two outgoing electron waves will interfere as in conventional diffraction. The figure indicates the phase difference between the two pathways. In the case of HHG, the wavelength of the generated light is many times larger than the bondlength of the molecule, so only the length difference in the incoming path, $\Delta_{1}$, contributes to the phase difference. For elastic scattering, both incoming and outgoing paths must be taken into account so that the total phase difference is proportional to $\Delta_{1}+\Delta_{2}$.

Clearly, the plane-wave model is a crude approximation because the electron wave packet becomes distorted as it approaches the nuclei. However, it has been demonstrated in Refs. [6, 8] that this model agrees remarkably well with fully numerical results if one uses for the evaluation of the phase difference an "effective"

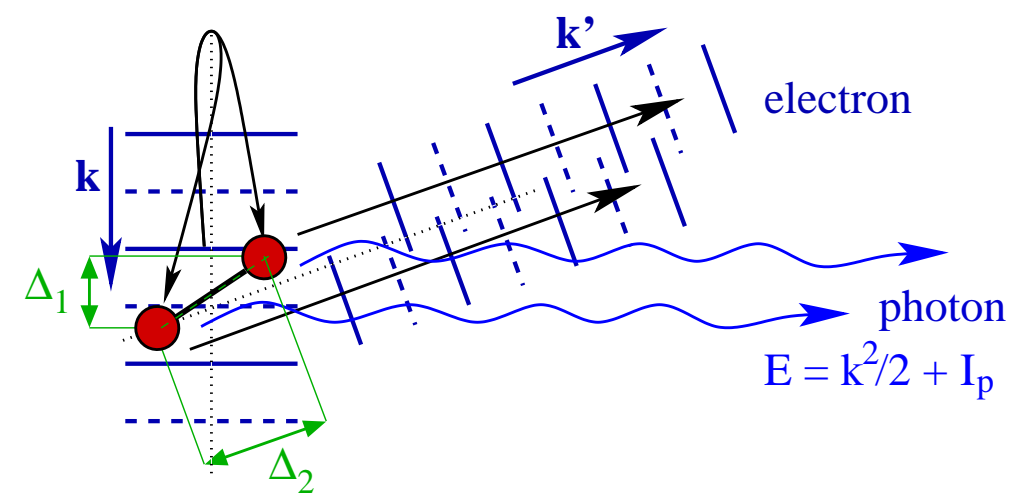

Figure 1: Illustration of laser-driven recollision in a two-centre molecule. 
wave vector $\mathbf{k}_{\mathrm{i}}$ defined by (atomic units are used throughout)

$$
\mathbf{k}_{\mathrm{i}}^{2} / 2=\mathbf{k}^{2} / 2+I_{\mathrm{p}}
$$

where $I_{\mathrm{p}}$ is the ionization potential. This definition approximately takes into account the increased electron momentum in the vicinity of the core, as compared to the asymptotic value $\mathbf{k}$ far from the core. The relevant phase differences for HHG and high-order ATI are then

$$
\begin{aligned}
(\Delta \phi)^{\mathrm{HHG}} & =k_{\mathrm{i}} \Delta_{1}, \\
(\Delta \phi)^{\mathrm{ATI}} & =k_{\mathrm{i}}\left(\Delta_{1}+\Delta_{2}\right) .
\end{aligned}
$$

In HHG, the energy $E_{\mathrm{ph}}$ of the emitted photon equals the return energy of the electron plus the binding energy. Therefore, we have a simple relationship between $k_{\mathrm{i}}$ and the measured photon energy,

$$
k_{\mathrm{i}}^{2} / 2=E_{\mathrm{ph}} .
$$

By inserting $k_{\mathrm{i}}$ into equation (2), the HHG phase difference is expressed in terms of the molecular geometry and the photon energy. In high-order ATI, the situation is more complicated because the electron is further accelerated after scattering. Therefore, one cannot give a closed expression for the phase difference in terms of the final electron momentum only [8].

To demonstrate the existence of these interference effects in HHG and ATI, we consider a two-dimensional and a three-dimensional model system describing a hydrogen molecular ion at its equilibrium internuclear distance $R=2$ a.u. The time-dependent Schrödinger equation for the case of a laser with linear polarization along the $z$-axis and time-dependent electric field $E(t)$ reads

$$
i \frac{\partial \Psi(\mathbf{r}, t)}{\partial t}=\left[\frac{\mathbf{p}^{2}}{2}+p_{z} A(t)+V(\mathbf{r})\right] \Psi(\mathbf{r}, t),
$$

where $A(t)=\int_{-\infty}^{t} E\left(t^{\prime}\right) d t^{\prime} . V(\mathbf{r})$ is the binding potential. In the 2D model, a widely used form of a soft-core potential is employed,

$$
V(\mathbf{r})=-\sum_{j=1,2} \frac{1}{\sqrt{\left(\mathbf{r}-\mathbf{R}_{j}\right)^{2}+0.5}},
$$

where $\mathbf{R}_{j}$ are the positions of the nuclei. In $3 \mathrm{D}$, in order to be able to reproduce the ionization potential of real $\mathrm{H}_{2}^{+}$, a different form of soft-core potential is used [7],

$$
V(\mathbf{r})=-\sum_{j=1,2} \frac{1}{\sqrt{0.1+\left(\mathbf{r}-\mathbf{R}_{j}\right)^{4} /\left(\left(\mathbf{r}-\mathbf{R}_{j}\right)^{2}+0.28\right)}} .
$$


The wave function is represented in cartesian coordinates on a grid with absorbing boundary. For the laser field, we assume trapezoidally shaped 10-cycle pulses (including three-cycle turn-on and turn-off times) with $780 \mathrm{~nm}$ wavelength. The time-dependent Schrödinger equation is solved numerically by means of the splitoperator method [15].

The complex amplitude for emission of harmonics polarized along $\hat{\mathbf{e}}$ is given by the Fourier transform of the dipole acceleration [16],

$$
A_{\hat{\mathbf{e}}}(\omega)=\int\langle\Psi(t)|\hat{\mathbf{e}} \cdot[\nabla V+\mathbf{E}(t)]| \Psi(t)\rangle e^{i \omega t} d t
$$

and the harmonic spectrum is obtained as

$$
S_{\hat{\mathbf{e}}}(\omega) \sim\left|A_{\hat{\mathbf{e}}}(\omega)\right|^{2}
$$

In a gaseous medium, only polarizations $\hat{\mathbf{e}}$ in the plane perpendicular to the laser propagation axis give rise to a phase-matched signal. Furthermore, the component parallel to the laser polarization is usually much stronger than the perpendicular component (see also the section on orientational phase matching).

Figure 2 displays a sample of harmonic spectra for different orientations of the molecule. The angle $\theta$ denotes the angle between the molecular axis and the electric field. Shown is the component polarized parallel to the laser field. We see that there is good agreement between 2D and 3D results. Clearly, interference minima are present in all spectra. The solid arrows indicate the positions of the minima deduced from the numerical results while the dashed arrows indicate those frequencies where destructive two-centre interference is expected from the plane-wave picture, i.e., where the phase difference in equation $(2)$ is $(\Delta \phi)^{\mathrm{HHG}}=\pi$. We note that there is good agreement between the numerically obtained interference structure and our two-centre interpretation, in particular for the 3D results.

From the preceding analysis, it is expected that the orientation dependence of HHG also exhibits interferences. This is confirmed in figure 3 where the phase and the modulus of the complex amplitude $A_{\hat{\mathbf{z}}}$ as a function of the molecular orientation are displayed for the $31^{\text {st }}$ harmonic in the $2 \mathrm{D}$ model. The different curves refer to three different laser intensities and are remarkably similar to each other. The harmonic phase undergoes a jump by about $\pi$ at a critical angle, and a minimum occurs in the amplitude at the same angle.

In the calculations described so far, the information about outgoing electrons is lost due to the absorbing boundary at the edge of the grid. In order to calculate photoelectron spectra, it is necessary to extend the numerical procedure. To this end, space is divided into an inner region (I) and an outer region (II). The absorbed wave packets are not discarded but coherently transferred from I to II, and the wave function $\Psi_{\mathrm{II}}$ is propagated under the influence of the laser only while the interaction 


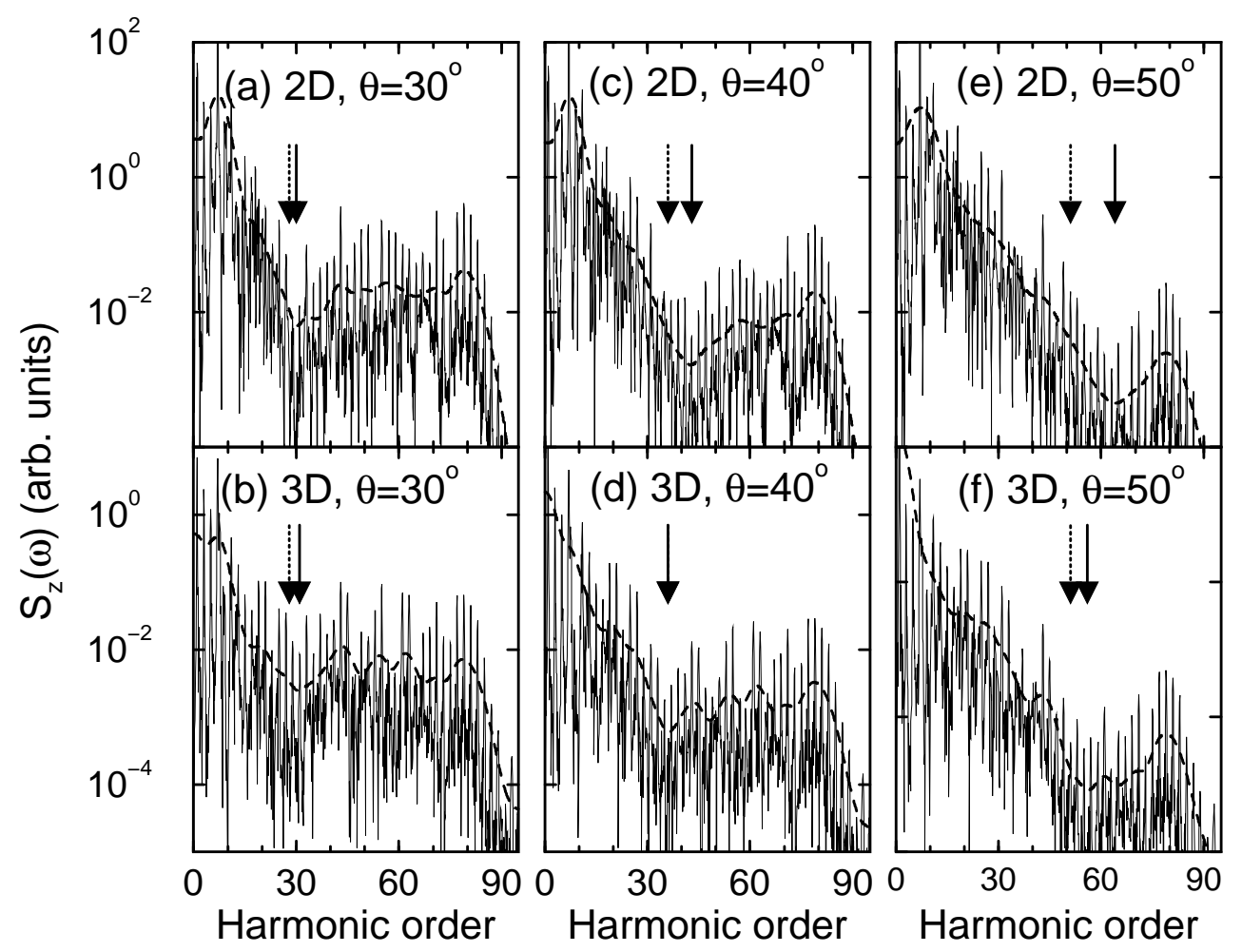

Figure 2: Calculated spectra of harmonics polarized parallel to the laser field for different orientations $\theta$ of the $\mathrm{H}_{2}^{+}$model molecule. The laser intensity is $5 \times 10^{14} \mathrm{~W} / \mathrm{cm}^{2}$. Explanation of arrows see text.

with the core is neglected [8],

$$
i \frac{\partial \Psi_{\mathrm{II}}(\mathbf{p}, t)}{\partial t}=\left[\frac{\mathbf{p}^{2}}{2}+p_{z} A(t)\right] \Psi_{\mathrm{II}}(\mathbf{p}, t) .
$$

Since $\Psi_{\mathrm{II}}$ is represented in momentum space, the ATI spectrum and the angular distribution are directly obtained from $\Psi_{\mathrm{II}}$ at the end of the propagation.

The upper panels of figure 4 show such numerically obtained angular distributions for the $2 \mathrm{D} \mathrm{H}_{2}^{+}$molecule aligned perpendicular to the laser field. The two distributions refer to the two electron energies $7 U_{\mathrm{p}}$ and $8 U_{\mathrm{p}}$, expressed in terms of the ponderomotive energy $U_{\mathrm{p}}=E_{0}^{2} /\left(4 \omega_{\mathrm{L}}^{2}\right)$. The two lower panels show the distributions obtained from a semiclassical model [8] that takes into account the two-centre interference with a phase difference given by equation (3). We note the excellent agreement between the two calculations. The distinct minima at $\pm 20^{\circ}$ on either side of the central maxima are not present in angular distributions for atoms [17, 18]. 


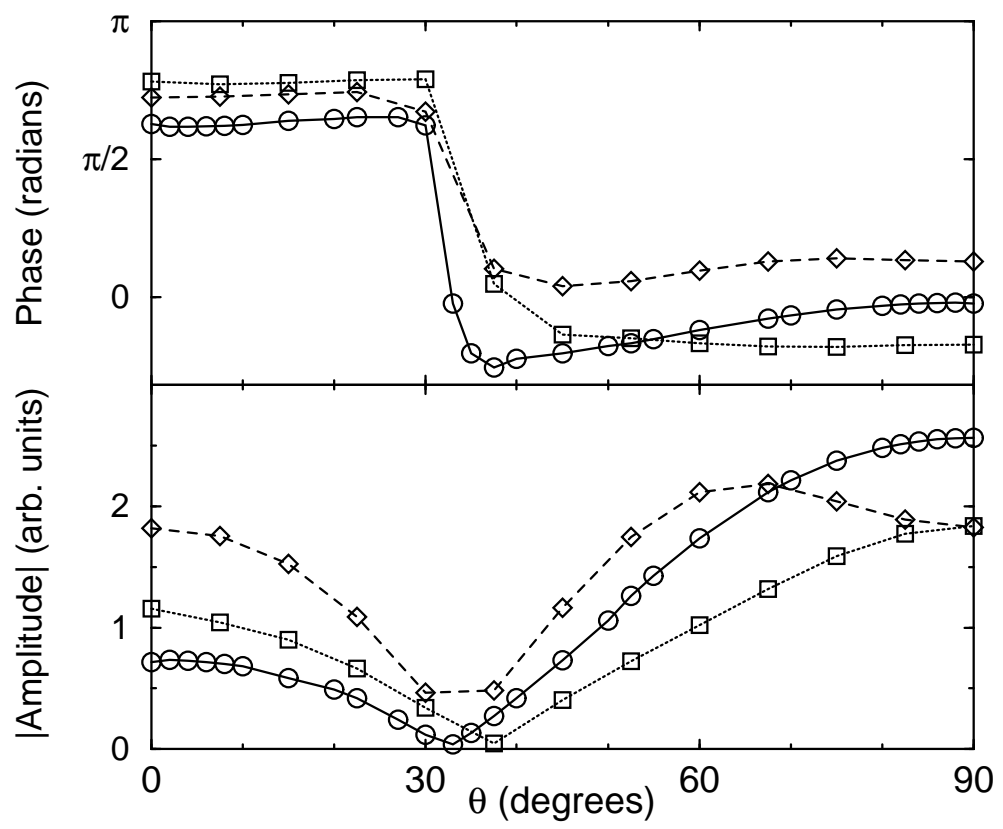

Figure 3: Calculated phase and modulus of the amplitude for the $31^{\text {st }}$ harmonic in $2 \mathrm{D}$ $\mathrm{H}_{2}^{+}$, polarization parallel to the laser field. The laser intensities are $5 \times 10^{14} \mathrm{~W} / \mathrm{cm}^{2}$ (circles), $7 \times 10^{14} \mathrm{~W} / \mathrm{cm}^{2}$ (squares) and $1 \times 10^{15} \mathrm{~W} / \mathrm{cm}^{2}$ (diamonds).

This structure is thus a diffraction pattern arising from the presence of two scatterers.

\section{Orientation dependence of high-harmonic generation}

For a clear appearance of interference effects due to molecular structure it is essential that the molecules are - at least to a certain degree - aligned along a given axis. The harmonic spectrum then depends on the orientation of this axis. In this section, we review our theoretical and experimental work on the orientation dependence for $\mathrm{CO}_{2}[12]$. New theoretical results including orientational phase matching follow in section 4 .

$\mathrm{CO}_{2}$ is a linear molecule and, similarly to the $\mathrm{O}_{2}$ molecule, the highest occupied molecular orbital (HOMO) is doubly degenerate and has antibonding $\pi_{\mathrm{g}}$ symmetry [19]. A possible representation of the two orbitals is such that they possess two nodal planes, one along the molecular axis and one perpendicular to it. This structure is interesting in the context of electron recollision since it has been argued that recollisions are suppressed when the laser field points along a nodal plane [20, 21]. This happens because the mirror antisymmetry forbids electrons with zero lateral 

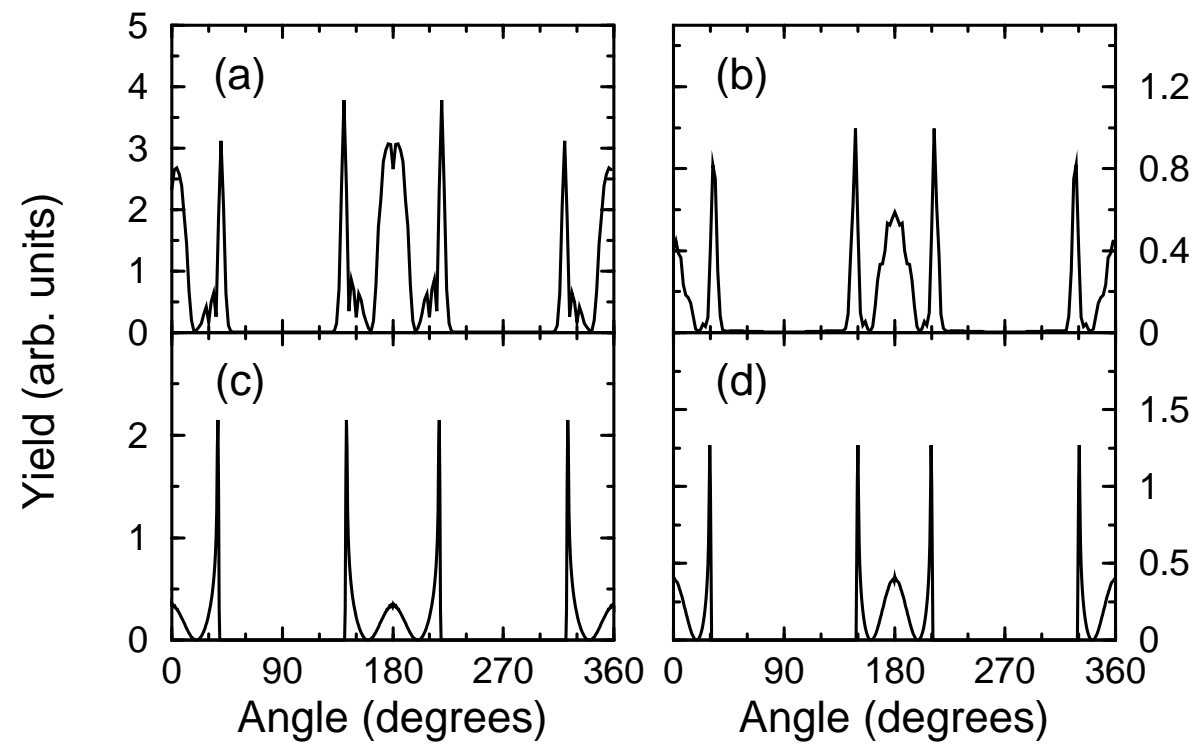

Figure 4: Angular distribution of ATI electrons for the 2D $\mathrm{H}_{2}^{+}$molecule aligned perpendicular to the field. Panels (a) and (b) show numerical quantum-mechanical results for electron energies of $7 U_{\mathrm{p}}$ and $8 U_{\mathrm{p}}$, respectively. (c) and (d) are the results of a semiclassical two-centre interference model [8]. The laser intensity is $5 \times 10^{14} \mathrm{~W} / \mathrm{cm}^{2}$.

velocity so that electrons tend to drift away from the polarization axis and eventually miss the core. For $\mathrm{CO}_{2}$, this should happen when the field is either parallel or perpendicular to the $\mathrm{CO}_{2}$ axis.

A two-dimensional model system that mimics the structure of $\mathrm{CO}_{2}$ has been employed to calculate the HHG orientation dependence. The model employs a three-centre soft-core potential and reproduces the ionization potential of $\mathrm{CO}_{2}$ and the twofold mirror antisymmetry of the valence orbital (see [12] for details). Here, $\sin ^{2}$-shaped 48-cycle pulses with $797 \mathrm{~nm}$ wavelength and $2 \times 10^{14} \mathrm{~W} / \mathrm{cm}^{2}$ peak intensity were assumed in order to compare with the experiment described below.

Figure 5(a) shows the calculated orientation dependence of the harmonic orders 9 to 17 . In all cases, the intensity is low for parallel and perpendicular alignment and peaks at intermediate angles. This behaviour is expected as a consequence of the twofold antisymmetry. Note the contrast to HHG in the simpler systems $\mathrm{H}_{2}$ and $\mathrm{H}_{2}^{+}$, where the ground-state orbitals are symmetric, and our previous calculations have shown that the harmonic yield is typically maximised for parallel and perpendicular alignment [5]. The interference effects discussed in section 2 are probably not observable at these relatively low harmonic orders where the plane-wave picture becomes rather inaccurate. The sharp peak at about $75^{\circ}$ seems to be due to a res- 


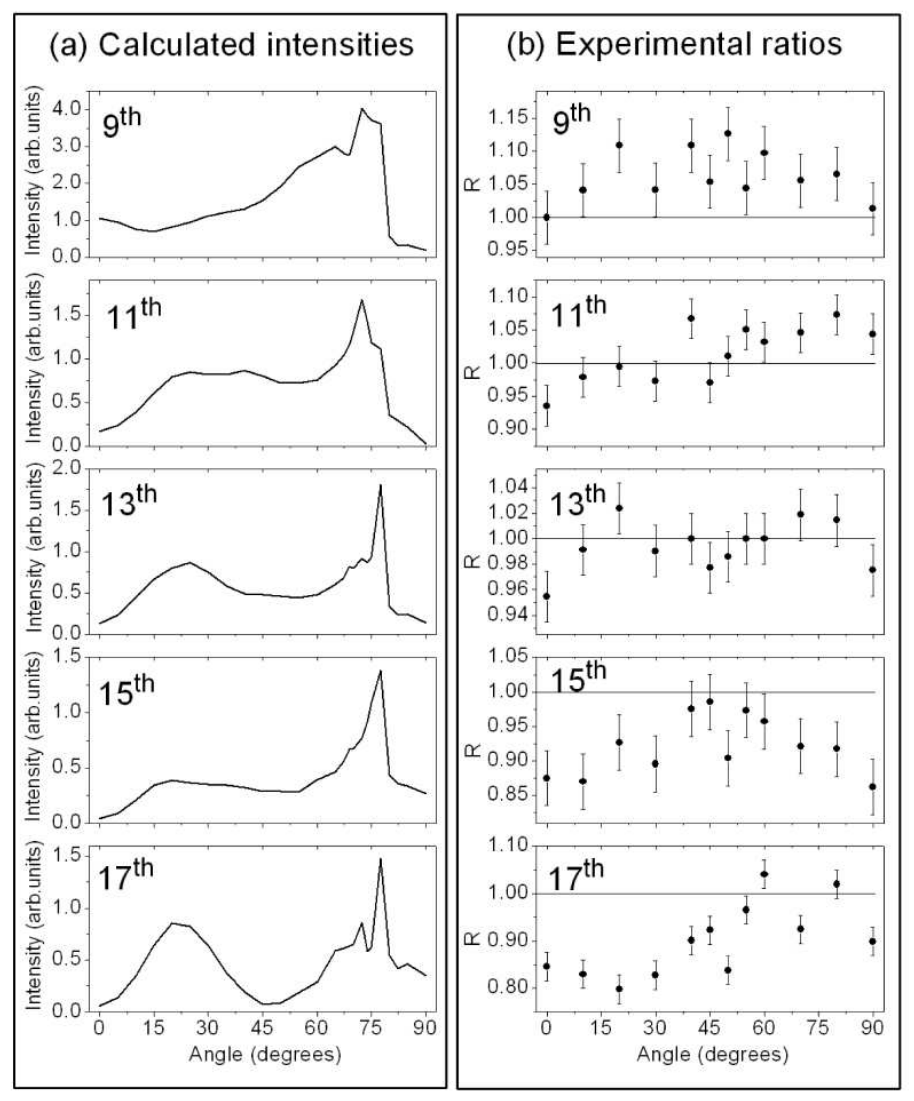

Figure 5: Orientation dependence of (a) the calculated harmonic intensities and (b) the measured ratios between an aligned and an isotropic ensemble for high-harmonic generation with $\mathrm{CO}_{2}$. The laser intensity is $2 \times 10^{14} \mathrm{~W} / \mathrm{cm}^{2}$.

onant enhancement of the ionization probability rather than due to the recollision dynamics [12].

Experimentally, control over the molecular orientation is required to measure the orientation dependence, and it is necessary to produce an aligned ensemble of sufficient density so that detectable harmonic emission is generated. Various methods, reviewed recently in reference [22], may be used. Here, we use the technique of adiabatic alignment with a strong off-resonant laser field, which has been successfully refined recently [23] to achieve high degrees of alignment. The adiabaticity condition is always satisfied in our experiments, since the preparation pulse, with 300 ps duration, is much longer than the rotational period of $\mathrm{CO}_{2}$ (43 ps). The effectiveness of the alignment technique is very sensitive to the initial rotational 


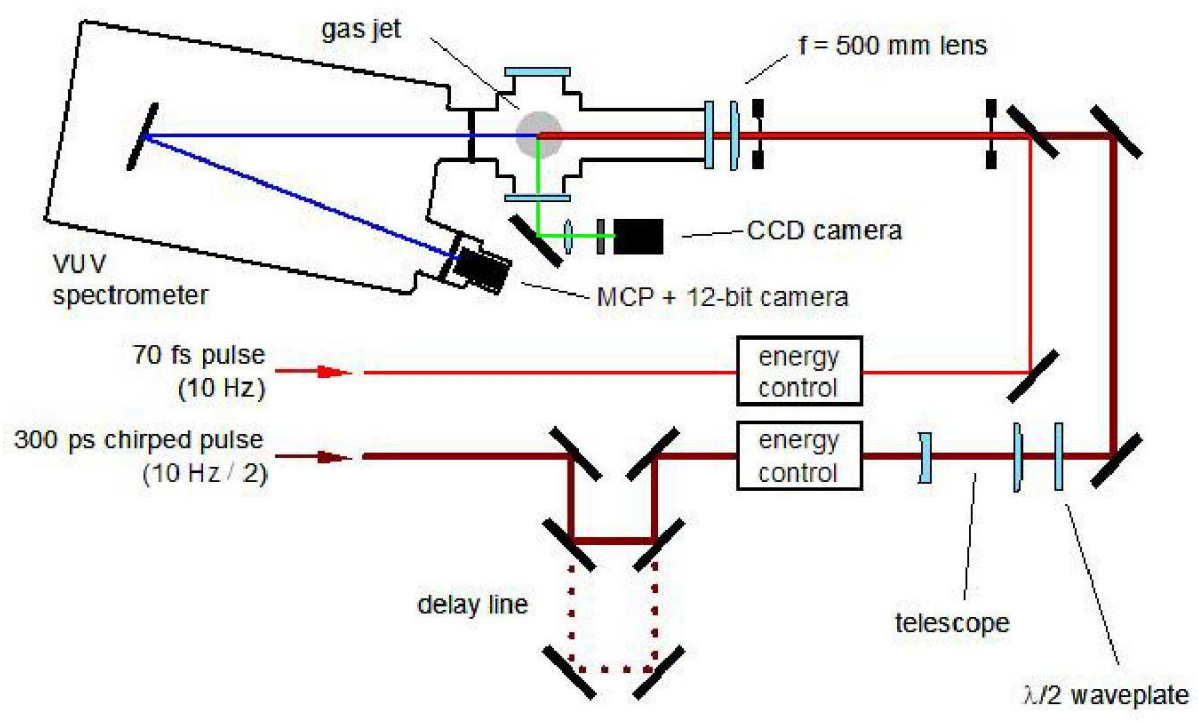

Figure 6: Experimental setup for high-harmonic generation with aligned $\mathrm{CO}_{2}$.

temperature of the ensemble, which in our case we estimate to be $25 \mathrm{~K}$ [24]. The degree of alignment can be quantified using the expectation value of $\cos ^{2} \vartheta^{\prime}$, where $\vartheta^{\prime}$ is the angle between the molecular axis and the aligning field. A perfectly aligned sample would give $\left\langle\cos ^{2} \vartheta^{\prime}\right\rangle=1$, whereas $\left\langle\cos ^{2} \vartheta^{\prime}\right\rangle=1 / 3$ corresponds to a randomly oriented ensemble. Using the results reported in [25], we estimate that the degree of alignment in the $\mathrm{CO}_{2}$ ensemble was characterised by $\left\langle\cos ^{2} \vartheta^{\prime}\right\rangle=0.55$ for a preparation beam intensity of $2 \times 10^{12} \mathrm{~W} / \mathrm{cm}^{2}$. With this moderate value we are limited in our ability to observe narrow features in the angular dependences, so the analysis must be restricted to the broad structures.

This aligned ensemble of $\mathrm{CO}_{2}$ molecules was studied as a source of high-order harmonic radiation. The experimental setup is schematically shown in figure 6 . The preparation pulse (alignment pulse) and the pump pulse (the pulse that causes the emission of harmonics) were generated in a Ti:sapphire chirped pulse amplification laser system with a centre wavelength of $797 \mathrm{~nm}$ and $60 \mathrm{~mJ}$ pulse energy working at $10 \mathrm{~Hz}$ repetition rate. A beam splitter immediately before the grating compressor divided the laser pulse into two parts. The reflected beam was compressed to produce pulses with 70 fs FWHM, and the transmitted fraction was left uncompressed to provide the 300 ps FWHM chirped preparation pulses. The preparation beam was modulated at $5 \mathrm{~Hz}$ by a mechanical chopper wheel in order to optimise the signal-to-noise ratio. The polarization direction of the preparation pulse, which fixes the axis of preferential alignment, could be varied smoothly with a half-wave 
plate while keeping the intensity constant. The pulses were recombined with a 50:50 beam splitter and copropagated through a $55 \mathrm{~cm}$ focal length lens into the interaction chamber, where a pulsed molecular jet, backed by a reservoir with $\mathrm{CO}_{2}$, was expanded perpendicularly to the laser propagation direction. The exit of the chamber was connected to the entrance slit of a vacuum ultra-violet spectrometer equipped with an imaging MCP detector on the exit port. The phosphor screen was imaged onto a 12-bit CCD camera interfaced with the data-acquisition computer. The horizontal dimension in the images contained the harmonic spectrum, so that several harmonics were detected simultaneously; spatial information was contained in the vertical dimension. For each data point we recorded two (200-shot averaged) images, only in one of which the preparation beam was present. Typically, the preparation pulse produced changes of the order of $10-30 \%$ in the HHG yield. We believe we can attribute this only moderate modulation, at least in part to the relatively low degree of alignment (compare also section 4).

For data analysis, the HHG spectra acquired when the preparation pulse was present were compared to those acquired when it was blocked by the chopper wheel. Vertical cross sections were taken across the emission region for each harmonic and the ratios of enhancement or suppression with respect to the isotropic ensemble in the centre of the emission profile were measured. These are the ratios $R$ shown in figure $5(\mathrm{~b})$, where the change in harmonic yield was studied as the alignment angle was slowly varied from $0^{\circ}$ to $90^{\circ}$ with respect to the pump polarization direction. Interestingly, not all harmonics show identical behaviour. The $11^{\text {th }}$ harmonic shows the greatest differences, the emission displaying suppression when the ensemble is preferentially aligned parallel to the field, but enhancement when it is aligned perpendicular to the field. The $17^{\text {th }}$ harmonic shows a similar behaviour: even though aligning the sample results mostly in suppression of the harmonic emission relative to the isotropic case, the situation where the molecular axis is aligned perpendicularly is much more favourable than parallel alignment. For the $9^{\text {th }}, 13^{\text {th }}$ and $15^{\text {th }}$ harmonics, the emission peaks at intermediate angles, and the general structure confirms what is expected for symmetry reasons, that is, local minima at $0^{\circ}$ and $90^{\circ}$. However, the errors associated with the measurement and the limited degree of alignment do not allow us to confirm or discard the existence of detailed structure for intermediate angles.

The observation of a reduced HHG yield when the molecular axis is parallel to the electric field, relative to other orientations - a feature that is common to all high harmonics observed - is contrary to recent findings in $\mathrm{N}_{2}$, but compatible with results for $\mathrm{O}_{2}$ [13]. This supports the idea that it is the antisymmetric nature of the $\mathrm{HOMO}$ orbitals in $\mathrm{CO}_{2}$ and $\mathrm{O}_{2}$ that causes the harmonic yield to maximise at angles other than $0^{\circ}$. In fact, this is one of the first indications that information about the molecular structure can be inferred from HHG. 


\section{Phase matching in an ensemble with different molecular orientations}

In an HHG experiment with an ensemble of molecules, we have a range of molecular orientations. Without aligning pulse, this is a uniform distribution over all possible angles. If the molecules are prealigned, their axes will lie preferentially inside a cone around the aligning-field direction. Since the contributions to the emission spectrum from the different orientations must be added coherently, the outcome depends crucially on the phases of the harmonics emitted from molecules in the different orientations. Even in the case of linear molecules, the theoretical treatment is somewhat cumbersome because the distribution is not cylindrically symmetric in the frame attached to the direction of the field $\mathbf{E}$ that generates the harmonics (frame $B$ ). We denote the angle between this field and the molecular axis as $\vartheta$, whereas $\vartheta^{\prime}$ is the angle between the molecule and the aligning field $\mathbf{E}^{\prime}$, i.e. the polar angle in the frame $B^{\prime}$ that is fixed to $\mathbf{E}^{\prime}$. Furthermore, we assume the geometry of the experiment described in section 3. This means that aligning and pump pulses have collinear propagation directions so that $\mathbf{E}$ and $\mathbf{E}^{\prime}$ are lying in a plane perpendicular to the beam axis.

The transformation between the two coordinate systems is illustrated in figure 7 . $\theta$ is the angle between the aligning field and the pump field. Thus, $\theta$ determines the orientation of the ensemble (as opposed to the orientation of an individual molecule).

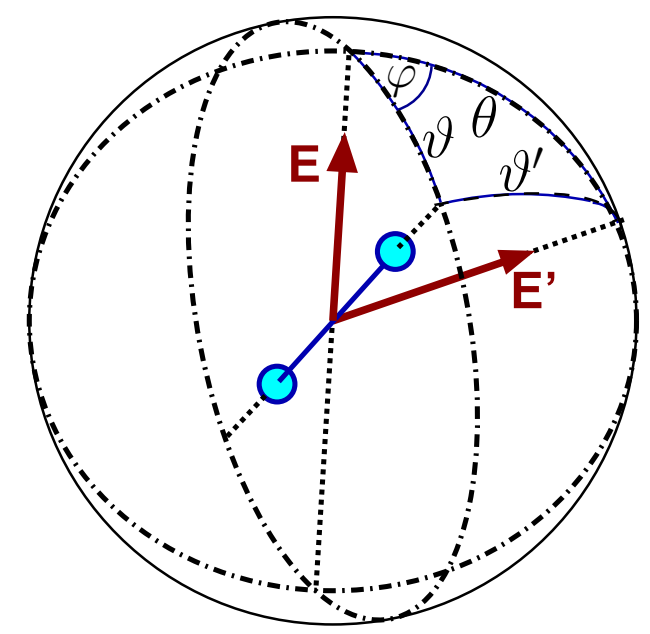

Figure 7: Transformation between the frames attached to the aligning field $\mathbf{E}^{\prime}$ and the pump field $\mathbf{E}(t)$. 
According to the cosine theorem of spherical geometry, the angles are related to each other by

$$
\cos \vartheta^{\prime}=\cos \vartheta \cos \theta+\sin \vartheta \sin \theta \cos \varphi,
$$

where $\varphi$ is the azimuthal angle of the molecular orientation in frame $B$. The aligning field creates a distribution of molecular axes that is cylindrically symmetric in frame $B^{\prime}$. It is denoted as $f_{0}\left(\vartheta^{\prime}\right)$ and shall be normalised such that

$$
\int_{0}^{2 \pi} \int_{0}^{\pi} f_{0}\left(\vartheta^{\prime}\right) \sin \vartheta^{\prime} d \vartheta^{\prime} d \varphi^{\prime}=1
$$

The transformation from $B$ to $B^{\prime}$ is a rotation that leaves the solid angle element invariant. Thus the distribution $f_{\theta}$ in frame $B$ is given by

$$
f_{\theta}(\vartheta, \varphi)=f_{0}\left(\vartheta^{\prime}(\vartheta, \varphi)\right)
$$

where the function $\vartheta^{\prime}(\vartheta, \varphi)$ is given by equation $(11)$.

In frame $B$, we take the laser propagation axis along $y$ and the pump field along $z$. The total harmonic yield for the ensemble is then the sum of the components along $z$ and $x$,

$$
\begin{aligned}
S_{\theta}(\omega) \sim & \left|\iint A_{x}(\vartheta, \varphi, \omega) f_{\theta}(\vartheta, \varphi) \sin \vartheta d \vartheta d \varphi\right|^{2} \\
+ & \left|\iint A_{z}(\vartheta, \varphi, \omega) f_{\theta}(\vartheta, \varphi) \sin \vartheta d \vartheta d \varphi\right|^{2}
\end{aligned}
$$

Since we work with linear molecules, the amplitudes $A_{z}$ and $A_{x}$ satisfy the symmetry properties

$$
\begin{aligned}
& A_{x}(\vartheta, \varphi, \omega)=A_{x}(\vartheta, \varphi=0, \omega) \cos \varphi \\
& A_{z}(\vartheta, \varphi, \omega)=A_{z}(\vartheta, \omega) .
\end{aligned}
$$

We can then write

$$
S_{\theta}(\omega) \sim\left|\int_{0}^{\pi} A_{x}(\vartheta, \varphi=0, \omega) g_{\theta}(\vartheta) \sin \vartheta d \vartheta\right|^{2}+\left|\int_{0}^{\pi} A_{z}(\vartheta, \omega) h_{\theta}(\vartheta) \sin \vartheta d \vartheta\right|^{2}
$$

with

$$
\begin{aligned}
g_{\theta}(\vartheta, \varphi) & =\int_{0}^{2 \pi} f_{\theta}(\vartheta, \varphi) \cos \varphi d \varphi, \\
h_{\theta}(\vartheta, \varphi) & =\int_{0}^{2 \pi} f_{\theta}(\vartheta, \varphi) d \varphi .
\end{aligned}
$$


We specialise now to symmetric linear molecules. In this case, the amplitudes satisfy the additional symmetry property

$$
A_{x / z}(\vartheta, \varphi, \omega)=A_{x / z}(\pi-\vartheta, \varphi+\pi, \omega)
$$

and the same relation holds for the distribution of orientations,

$$
f_{\theta}(\vartheta, \varphi, \omega)=f_{\theta}(\pi-\vartheta, \varphi+\pi, \omega) .
$$

Hence, the integrations in equation (18) are simplified,

$$
S_{\theta}(\omega) \sim\left|2 \int_{0}^{\pi / 2} A_{x}(\vartheta, \varphi=0, \omega) g_{\theta}(\vartheta) \sin \vartheta d \vartheta\right|^{2}+\left|2 \int_{0}^{\pi / 2} A_{z}(\vartheta, \omega) h_{\theta}(\vartheta) \sin \vartheta d \vartheta\right|^{2} .
$$

For randomly oriented molecules, we have $f_{\theta} \equiv 1 / 4 \pi, g_{\theta} \equiv 0$ and $h_{\theta} \equiv 1 / 2$, and therefore,

$$
S_{\theta}^{\text {random }} \sim\left|\int_{0}^{\pi / 2} A_{z}(\vartheta, \omega) \sin \vartheta d \vartheta\right|^{2}
$$

i.e., all harmonics are polarized parallel to the generating laser field.

It is a considerable task in itself to calculate the distribution function following laser-induced alignment exactly, and this shall not be the main point here. Rather, we make a simple ansatz in order to study the effect of imperfect alignment on the harmonics. We assume

$$
f_{0}\left(\vartheta^{\prime}\right)=\frac{\beta}{2 \pi \ln \frac{\beta+1}{\beta-1}} \frac{1}{\beta^{2}-\cos ^{2} \vartheta^{\prime}}
$$

where the parameter $\beta>1$ is related to the degree of alignment as

$$
\left\langle\cos ^{2} \vartheta^{\prime}\right\rangle=\beta^{2}-\frac{2 \beta}{\ln \frac{\beta+1}{\beta-1}}
$$

and the limit $\beta \rightarrow 1$ corresponds to perfect alignment. This choice of $f_{0}$ has the advantage that the integrals in equations (19) and (20) can be evaluated analytically.

We have calculated the HHG spectra for the $2 \mathrm{D}$ model of $\mathrm{CO}_{2}$ using the distribution (25) with $\beta=1.04$ (corresponding to $\left\langle\cos ^{2} \vartheta^{\prime}\right\rangle=0.55$ ) and $\beta=1.0082$ (corresponding to $\left\langle\cos ^{2} \vartheta^{\prime}\right\rangle=0.65$ ). We have then taken the ratio compared to the case of random alignment. The resulting orientation dependences are shown in figure 8 for the harmonics 9 to 17 . Clearly, the curves approach the limit of random orientation $(R \equiv 1)$ when the width of the distribution is increased. We see that for $\left\langle\cos ^{2} \vartheta^{\prime}\right\rangle=0.55$, the ratio $R$ takes values between about 0.4 and 2.3. This 


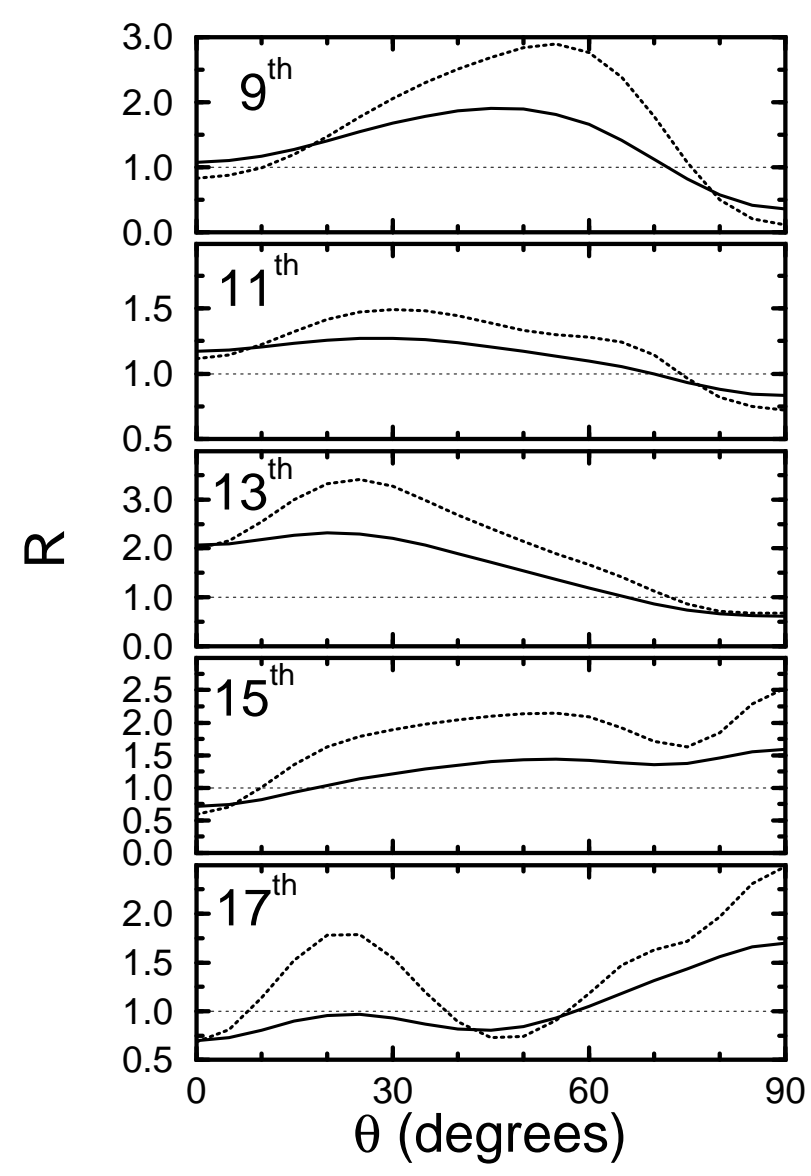

Figure 8: Calculated orientation dependence of harmonic intensities for the $\mathrm{CO}_{2}$ molecule for distributions with different degrees of alignment. Solid, $\left\langle\cos ^{2} \vartheta^{\prime}\right\rangle=0.55$; dashed, $\left\langle\cos ^{2} \vartheta^{\prime}\right\rangle=0.65$.

range overestimates somewhat the experimental range (0.8-1.15), and the discrepancy should be due to phase-matching effects in the propagation of the harmonics, which are not taken into account here. As compared to the calculated intensities shown in figure 5 where the yield was always suppressed at $\theta=90^{\circ}$, this minimum is now smeared out and has even disappeared for the $15^{\text {th }}$ and $17^{\text {th }}$ harmonic. The resulting orientation dependence is similar to the experimental ones observed for the $11^{\text {th }}$ and $17^{\text {th }}$ harmonic.

We conclude that the inclusion of orientational phase matching represents a major step forward in the theoretical analysis. It is also important to recognise the role of the orientation dependent phase. This is an additional source of phase mismatch compared to HHG with atoms. 


\section{Conclusions}

Our theoretical predictions for HHG and high-order ATI with aligned molecules provide a very promising perspective for applications to ultrafast imaging of molecules. In the light of recent advances in experimental alignment techniques, we are optimistic that clear signatures of two-centre interferences will be observed in future experiments. As a first step towards this goal, we have measured the orientation dependence of $\mathrm{HHG}$ in $\mathrm{CO}_{2}$ and have shown that the results are in agreement with the predictions made for the twofold antisymmetry of the $\mathrm{CO}_{2}$ valence orbital. Although the observed effects are not related to the diffraction-type interference as described in section 2, they are a first demonstration that the structure of the molecule imprints its signatures on the orientation dependence of the harmonics. We have given a guideline to the calculation of HHG with imperfectly aligned molecules, and we have shown that orientational phase matching plays an important role in HHG with molecules.

\section{Acknowledgements}

We gratefully acknowledge useful discussions with Drs. V. Herrero, J. W. G. Tisch, K. J. Mendham, E. Sali and O. Rubner and the technical assistance of P. Ruthven and A. Gregory. The experimental work was supported by the U.K. Engineering and Physical Sciences Research Council (Grant No. GR/N11292) and MOD under the joint support scheme. Furthermore, we acknowledge the support by the European Union "COCOMO" network (EU-IHP HPRN-CT-1999-00129) and by the British-Spanish Acciones Integradas Scheme.

\section{References}

[1] Kienberger, R., Goulielmakis, E., Uiberacker, M., Baltuska, A., Yakovlev, V., Bammer, F., Scrinzi, A., Westerwalbesloh, T., Kleineberg, U., Heinzmann, U., Drescher, M., and Krausz, F., 2004, Nature, 427, 817.

[2] Nimkura, H. , Légare, F., Hasbani, R., Bandrauk, A. D., Ivanov, M. Yu., Villeneuve, D. M., and Corkum, P. B., 2003, Nature, 421, 826.

[3] Corkum, P. B., 1993, Phys. Rev. Lett., 71, 1994.

[4] Paulus, G. G., Nicklich, W., Huale, X., Lambropoulos, P., and Walther, H., 1994, Phys. Rev. Lett., 72, 2851. 
[5] Lein, M., Hay, N., Velotta, R., Marangos, J. P., and Knight, P. L., 2002, Phys. Rev. Lett., 88, 183903.

[6] Lein, M., Hay, N., Velotta, R., Marangos, J. P., and Knight, P. L., 2002, Phys. Rev. A, 66, 023805.

[7] Lein, M., Corso, P. P., Marangos, J. P., and Knight, P. L., 2003, Phys. Rev. A, 67, 023819.

[8] Lein, M., Marangos, J. P., and Knight, P. L., 2002, Phys. Rev. A, 66, 051404(R).

[9] Muth-Böhm, J., Becker, A., and Faisal, F. H. M., 2000, Phys. Rev. Lett., 85, 2280.

[10] Yu, H., and Bandrauk, A. D., 1995, J. Chem. Phys., 102, 1257; Kopold, R., Becker, W., and Kleber, M., 1998, Phys. Rev. A, 58, 4022; Lappas, D. G., and Marangos, J. P., 2000, J. Phys. B, 33, 4679.

[11] Velotta, R., Hay, N., Mason, M. B., Castillejo, M., and MaranGos, J. P., 2001, Phys. Rev. Lett., 87, 183901.

[12] De Nalda, R., Heesel, E., Lein, M., Hay, N., Velotta, R., Springate, E., Castillejo, M., and Marangos, J. P., 2004, Phys. Rev. A, 69, 031804(R).

[13] Zeidler, D., Levesque, J., Itatani, J., Lee, K. F., Dooley, P. W., Litvinyuk, I. V., Villeneuve, D. M., and Corkum, P. B., Controlling high-harmonic generation via molecular alignment, to be published.

[14] Hay, N., Velotta, R., Lein, M., de Nalda, R., Heesel, E., Castillejo, M., and Marangos, J. P., 2002, Phys. Rev. A, 65, 053805.

[15] Feit, M. D., Fleck, J. A., Jr., and Steiger, A., 1982, J. Comput. Phys., 47,412 .

[16] Burnett, K., Reed, V. C., Cooper, J., and Knight, P. L., 1992, Phys. Rev. A, 45, 3347.

[17] Paulus, G. G., Becker, W., Nicklich, W., and Walther, H., 1994, J. Phys. B, 27, L703.

[18] Becker, W., Lohr, A., and Kleber, M., 1994, J. Phys. B, 27, L325.

[19] Jorgensen, W. L., and SAlem, L., The organic chemist's book of orbitals, (Academic Press, New York, 1973). 
[20] Bhardwaj, V. R., Rayner, D. M., Villeneuve, D. M., and Corkum, P. B., 2001, Phys. Rev. Lett., 87, 253003.

[21] Lein, M., 2003, J. Phys. B, 36, L155.

[22] Stapelfeldt, H., and Seideman, T., 2003, Rev. Mod. Phys., 75, 543.

[23] Sakai, H., Safvan, C. P., Larsen, J. J., Hilligscee, K. M., Hald, K., and Stapelfeldt, H., 1999, J. Chem. Phys., 110, 10235; Larsen, J. J., Sakai, H., Safvan, C. P., Wendt-Larsen, I., and Stapelfeldt, H., 1999, J. Chem. Phys., 111, 7774.

[24] Miller, D. R., in Atomic and molecular beam methods, edited by G. Scoles (Oxford University Press, New York, 1988), Vol. 1.

[25] Friedrich, B., and Herschbach, D., 1995, Phys. Rev. Lett., 74, 4623. 